\title{
THE CHOICE OF THE FORMS OF LYAPUNOV FUNCTIONS FOR A POSITIVE 2D ROESSER MODEL
}

\author{
TADEUSZ KACZOREK
}

\author{
Institute of Control and Industrial Electronics \\ Warsaw University of Technology \\ ul. Koszykowa 75, 00-662 Warsaw, Poland \\ e-mail: kaczorekeisep.pw.edu.pl
}

\begin{abstract}
The appropriate choice of the forms of Lyapunov functions for a positive 2D Roesser model is addressed. It is shown that for the positive 2D Roesser model: (i) a linear form of the state vector can be chosen as a Lyapunov function, (ii) there exists a strictly positive diagonal matrix $P$ such that the matrix $A^{T} P A-P$ is negative definite. The theoretical deliberations will be illustrated by numerical examples.
\end{abstract}

Keywords: Lyapunov function, positive 2D Roesser model, asymptotic stability

\section{Introduction}

The most popular models of two-dimensional (2D) systems are models introduced by Roesser (1975), Fornasini and Marchesini (1978; 1976), and Kurek (1985). The positive (nonnegative) 2D Roesser type model was introduced in (Kaczorek, 1996). Some recent developments in 2D positive systems theory were reported in (Fornasini and Valcher, 1996; Fornasini and Valcher, 1997; Valcher and Fornasini, 1995; Kaczorek, 2000). Benvenuti and Farina in (2004) showed that a positive asymptotically stable system is diagonally equivalent to a compartmental system.

The controllability of 2-D standard and positive linear systems was considered in (Bose, 1985; Klamka, 1991; Klamka, 1999; Kaczorek, 1985; Kaczorek, 1996; Kaczorek, 2000). The stability of the positive 2D Roesser model was investigated in (Kurek, 2002) and the asymptotic behaviour of 2D positive systems was analyzed in (Valcher and Fornasini, 1995).

In this paper the problem of the choice of Lyapunov functions for positive 2D linear systems described by the Roesser model will be addressed. It will be shown that for positive $2 \mathrm{D}$ linear systems a linear form of the state vector can be chosen as a Lyapunov function and there exists a strictly positive diagonal matrix $P$ such that the matrix $A^{T} P A-P$ is negative definite, where $A$ is a matrix of the 2D Roesser model. To the best of the author's knowledge, the choice of the forms of Lyapunov functions for the positive 2D Roesser model has not been considered yet.

\section{Preliminaries}

Let $\mathbb{Z}_{+}$be the set of nonnegative integers. The set of $n \times m$ real matrices with nonnegative entries will be denoted by $\mathbb{R}_{+}^{n \times m}$ and $\mathbb{R}_{+}^{n}=\mathbb{R}_{+}^{n \times 1}$. A matrix $A=\left[a_{i j}\right] \in \mathbb{R}^{n \times m}$ will be called strictly positive, which will be denoted by $A>0$, if and only if $a_{i j}>0$ for all $i=1, \ldots, n$ and $j=1, \ldots, m$. Similarly, a vector $x \in \mathbb{R}_{+}^{n}$ will be called strictly positive (resp. negative), which will be denoted by $x>0$ (resp. $x<0)$, if and only if all its components are positive (resp. negative).

Consider the positive discrete-time linear system

$$
x_{i+1}=A x_{i}+b u_{i}, \quad i \in \mathbb{Z}_{+},
$$

where $x_{i} \in \mathbb{R}_{+}^{n}$ and $u_{i} \in \mathbb{R}_{+}$are respectively the state and input vectors, and $A \in \mathbb{R}_{+}^{n \times n}, b \in \mathbb{R}_{+}^{n}$.

Let a constant input $\bar{u}>0$ be applied to the system (1). A vector $x_{e} \in \mathbb{R}_{+}^{n}$ is called an equilibrium state of the asymptotically stable system (1) if and only if

$$
x_{e}=A x_{e}+b \bar{u} .
$$

If the system (1) is asymptotically stable, then the matrix $\left[I_{n}-A\right]$ ( $I_{n}$ stands for the identity matrix) is invertible and $\left[I_{n}-A\right]^{-1} \in \mathbb{R}_{+}^{n \times n}$. From (2) we have

$$
x_{e}=\left[I_{n}-A\right]^{-1} b \bar{u} .
$$

It is well known (Farina and Rinaldi, 2000; Kaczorek, 2000) that if $b \bar{u}>0$, then $x_{e}>0$. Let $b \bar{u}=\mathbf{1}_{n}$, 
where $\mathbf{1}_{n}=[1, \ldots, 1]^{T}$ (' $\mathrm{T}$ ' denotes the transpose). Then from (3) we obtain

$$
\bar{x}_{e}=\left[I_{n}-A\right]^{-1} \mathbf{1}_{n}>0 .
$$

\section{Asymptotic Stability}

Consider the positive discrete-time linear system

$$
x_{i+1}=A x_{i}, \quad i \in \mathbb{Z}_{+},
$$

where $x_{i} \in \mathbb{R}_{+}$and $A \in \mathbb{R}_{+}^{n \times n}$.

Theorem 1. The positive discrete-time system (5) is asymptotically stable if and only if there exists a strictly positive vector $\bar{x}>0$ (i.e. $\bar{x} \in \mathbb{R}_{+}^{n}$ ) such that

$$
\left[A-I_{n}\right] \bar{x}<0 .
$$

Proof. If the system (5) is asymptotically stable, then we may choose $\bar{x}=\bar{x}_{e}=\left[I_{n}-A\right]^{-1} \mathbf{1}_{n}>0$, and from (6) we have

$$
\left[A-I_{n}\right] \bar{x}=\left[A-I_{n}\right]\left[I_{n}-A\right]^{-1} \mathbf{1}_{n}=-\mathbf{1}_{n}<0 .
$$

If there exists a vector $\bar{x}>0$ satisfying the condition (6), then as a Lyapunov function we may choose the linear form

$$
V\left(x_{i}\right)=\bar{x}^{T} x_{i}
$$

which is positive for every $x_{i} \in \mathbb{R}_{+}^{n}$ (i.e. $x_{i} \neq 0$ ).

Note that the dual system $x_{i+1}=A^{T} x_{i}$ is positive and asymptotically stable if and only if the original system (5) is positive and asymptotically stable.

From (8) and (5) we have

$$
\begin{aligned}
\Delta V\left(x_{i}\right)= & V\left(x_{i+1}\right)-V\left(x_{i}\right) \\
= & \bar{x}^{T}\left(x_{i+1}-x_{i}\right)=\bar{x}^{T}\left(A-I_{n}\right) x_{i}<0 \\
& \quad \text { for } x_{i} \in \mathbb{R}_{+}^{n}\left(x_{i} \neq 0\right)
\end{aligned}
$$

since by (6) we get $\bar{x}^{T}\left[A^{T}-I_{n}\right]<0_{n}^{T}=[0, \ldots, 0]$.

Collorary 1. As a Lyapunov function for the positive discrete-time linear system (5) we may choose the linear form (8).

\section{Positive 2D Roesser Model}

Consider the positive 2D Roesser model (Kaczorek, 1985; Kaczorek, 2000; Roesser, 1975)

$$
\begin{aligned}
{\left[\begin{array}{l}
x_{i+1, j}^{h} \\
x_{i, j+1}^{v}
\end{array}\right]=} & {\left[\begin{array}{ll}
A_{11} & A_{12} \\
A_{21} & A_{22}
\end{array}\right]\left[\begin{array}{l}
x_{i j}^{h} \\
x_{i j}^{v}
\end{array}\right] } \\
& +\left[\begin{array}{l}
B_{1} \\
B_{2}
\end{array}\right] u_{i j}, \quad i, j \in \mathbb{Z}_{+},
\end{aligned}
$$

where $x_{i j}^{h} \in \mathbb{R}_{+}^{n_{1}}$ and $x_{i j}^{v} \in \mathbb{R}_{+}^{n_{2}}$ are respectively the horizontal and vertical state vectors at the point $(i, j) \in$ $\mathbb{Z}_{+} \times \mathbb{Z}_{+}, u_{i j} \in \mathbb{R}_{+}^{m}$ is the input vector and $A_{k l} \in$ $\mathbb{R}_{+}^{n_{k} \times n_{l}}, B_{k} \in \mathbb{R}_{+}^{n_{k} \times m}, k, l=1,2$. Using the notation

$$
\begin{aligned}
x_{i j}^{\prime} & =\left[\begin{array}{l}
x_{i+1, j}^{h} \\
x_{i, j+1}^{v}
\end{array}\right], \quad x_{i j}=\left[\begin{array}{l}
x_{i j}^{h} \\
x_{i j}^{v}
\end{array}\right], \\
A & =\left[\begin{array}{ll}
A_{11} & A_{12} \\
A_{21} & A_{22}
\end{array}\right], B=\left[\begin{array}{l}
B_{1} \\
B_{2}
\end{array}\right],
\end{aligned}
$$

we may write (10) in the form

$$
x_{i j}^{\prime}=A x_{i j}+B u_{i j} .
$$

Let a constant input vector $\bar{u}>0\left(u_{i j}=\bar{u} \in\right.$ $\mathbb{R}_{+}^{m}, i, j \in \mathbb{Z}_{+}$) be applied to the positive $2 \mathrm{D}$ asymptotically stable Roesser model (10). Then $x_{e} \in \mathbb{R}_{+}^{n}$, where $n=n_{1}+n_{2}$, is called an equilibrium state of the model if and only if

$$
x_{e}=A x_{e}+B \bar{u} .
$$

If the model (10) is asymptotically stable, then the matrix $\left[I_{n}-A\right]$ is invertible and from (13) for $B \bar{u}>0$ we obtain (Kaczorek, 2002)

$$
x_{e}=\left[I_{n}-A\right]^{-1} B \bar{u}>0 .
$$

If $B \bar{u}=\mathbf{1}_{n}$, then from (14) we have

$$
\bar{x}_{e}=\left[I_{n}-A\right]^{-1} \mathbf{1}_{n}>\mathbf{1}_{n}
$$

since $\bar{x}_{e}=A \bar{x}_{e}+\mathbf{1}_{n}$ and $A \in \mathbb{R}_{+}^{n \times n}$.

Theorem 2. The positive $2 D$ Roesser model (10) is asymptotically stable if and only if there exists a strictly positive vector $\bar{x}>0\left(\bar{x} \in \mathbb{R}_{+}^{n}, \bar{x} \neq 0\right)$ such that

$$
\left[A-I_{n}\right] \bar{x}<0 .
$$

Proof. If the model (10) is asymptotically stable, then we may choose $\bar{x}=\bar{x}_{e}=\left[I_{n}-A\right]^{-1} \mathbf{1}_{n}>0$ and from (15) we obtain

$$
\left[A-I_{n}\right] \bar{x}=\left[A-I_{n}\right]\left[I_{n}-A\right]^{-1} \mathbf{1}_{n}=-\mathbf{1}_{n}<0 .
$$

Therefore, if the model (10) is asymptotically stable, then there exists a strictly positive vector $\bar{x}=\bar{x}_{e}$ satisfying the condition (16). If there exists a strictly positive vector $\bar{x}>0$ satisfying the condition (16), then as a Lyapunov function we may choose the linear form

$$
V\left(x_{i j}\right)=\bar{x}^{T} x_{i j},
$$

which is positive for every nonzero $x_{i j} \in \mathbb{R}_{+}^{n}$.

Note that the dual 2D model

$$
x_{i j}^{\prime}=A^{T} x_{i j}, \quad i, j \in \mathbb{Z}_{+},
$$


is positive and asymptotically stable if and only if the original 2D model (12) is positive and asymptotically stable.

From (18) and (12) we have

$$
\begin{aligned}
\Delta V\left(x_{i j}\right) & =V\left(x_{i j}^{\prime}\right)-V\left(x_{i j}\right) \\
& =\bar{x}^{T}\left(x_{i j}^{\prime}-x_{i j}\right) \\
& =\bar{x}^{T}\left(A-I_{n}\right) x_{i j}<0 \\
& \quad \text { for } x_{i j} \in \mathbb{R}_{+}^{n}\left(x_{i j} \neq 0\right)
\end{aligned}
$$

since by (15) we get $\bar{x}^{T}\left[A^{T}-I_{n}\right]<0_{n}^{T}$. This completes the proof.

Collorary 2. As a Lyapunov function for the positive $2 D$ Roesser model (10) we may choose the linear form (18).

Example 1. Consider the positive 2D Roesser model (10) with the matrices

$$
\begin{aligned}
& A=\left[\begin{array}{ll}
A_{11} & A_{12} \\
A_{21} & A_{22}
\end{array}\right]=\left[\begin{array}{cccc}
0.1 & a_{12} & \vdots & a_{13} \\
0 & 0.2 & \vdots & a_{23} \\
\ldots & \ldots & \ldots \\
0 & 0 & \vdots & a_{33}
\end{array}\right], \\
& B=\left[\begin{array}{l}
B_{1} \\
B_{2}
\end{array}\right]=\left[\begin{array}{c}
1 \\
1 \\
\ldots \\
1
\end{array}\right]
\end{aligned}
$$

for $a_{12} \geq 0, a_{13} \geq 0, a_{23} \geq 0, a_{33} \geq 0$. In this case

$$
\begin{aligned}
& {\left[I_{n}-A\right]^{-1}=\frac{1}{0.72\left(1-a_{33}\right)}} \\
& \quad \times\left[\begin{array}{ccc}
0.8\left(1-a_{33}\right) & a_{12}\left(1-a_{33}\right) & 0.8 a_{13}+a_{12} a_{23} \\
0 & 0.9\left(1-a_{33}\right) & 0.9 a_{23} \\
0 & 0 & 0.72
\end{array}\right] .
\end{aligned}
$$

If $a_{33}<1$, the model with (21) is asymptotically stable and the sum of every row of the matrix (22) is positive.

For $\bar{u}=1$ from (15) and (22) we have the equilibrium state of the system

$$
\begin{aligned}
\bar{x}_{e} & =\left[I_{n}-A\right]^{-1} 1_{n}=\frac{1}{0.72\left(1-a_{33}\right)} \\
& \times\left[\begin{array}{c}
\left(1-a_{33}\right)\left(0.8+a_{12}\right)+0.8 a_{13}+a_{12} a_{23} \\
0.9\left(1+a_{23}-a_{33}\right) \\
0.72
\end{array}\right] .
\end{aligned}
$$

As a Lyapunov function, in this case we may choose the linear form

$$
\begin{aligned}
V\left(x_{i j}\right)= & -\bar{x}_{e}^{T} x_{i j} \\
= & {\left[\left(1-a_{33}\right)\left(0.8+a_{12}\right)+0.8 a_{13}\right.} \\
& \left.+a_{12} a_{23}, 0.9\left(1+a_{23}-a_{33}\right)\right] x_{i j}^{h}+0.72 x_{i j}^{v}
\end{aligned}
$$

\section{Diagonal Form of the Matrix Defining a Lyapunov Function}

It is well known (Kaczorek, 2000) that the positive 2D Roesser model (10) is asymptotically stable if and only if there exists a positive definite matrix $P \in \mathbb{R}^{n \times n}$ such that

$$
A^{T} P A-P<0
$$

where the notation $M<0$ means that the matrix $M$ is negative definite.

In what follows we shall demonstrate that for the positive 2D Roesser model the positive definite matrix $P$ satisfying (23) can be chosen in a diagonal form.

Theorem 3. The positive 2D Roesser model (10) is asymptotically stable if and only if there exists a strictly positive diagonal matrix $P$ such that (23) holds.

Proof. If the positive 2D Roesser model is asymptotically stable, then from (15) it follows that its equilibrium state satisfies the equation

$$
\bar{x}_{e}=A \bar{x}_{e}+\mathbf{1}_{n}
$$

or

$$
\bar{x}_{e i}=a_{i} \bar{x}_{e}+1, i=1, \ldots, n,
$$

where $a_{i}$ is the $i$-th row of the matrix $A$ and $\bar{x}_{e}=$ $\left[\bar{x}_{e 1}, \bar{x}_{e 2}, \ldots, \bar{x}_{e n}\right]^{T}$.

Taking into account that $\bar{x}_{e i}>0$ for $i=1, \ldots, n$, we choose the strictly positive diagonal matrix $P$ in the form

$$
P=P_{1}^{T} P_{1}
$$

where

$$
P_{1}=\operatorname{diag}\left[\bar{x}_{e 1}, \bar{x}_{e 1}, \ldots, \bar{x}_{e n}\right] .
$$

From (25) and (27) we have

$$
\begin{aligned}
P_{1}=\text { blockdiag } & {\left[a_{1}, a_{2}, \ldots, a_{n}\right] } \\
\times \operatorname{blockdiag}[ & \left.\bar{x}_{e}, \bar{x}_{e}, \ldots, \bar{x}_{e}\right]+I_{n}
\end{aligned}
$$

and

$$
\begin{array}{r}
P_{1} x=\left[\left(a_{1} \bar{x}_{e}+1\right) x_{1},\left(a_{2} \bar{x}_{e}+1\right) x_{2}, \ldots,\right. \\
\left.\left(a_{n} \bar{x}_{e}+1\right) x_{n}\right]^{T},
\end{array}
$$

where $x_{i}(i=1, \ldots, n)$ is the $i$-th component of the vector $x$.

Postmultiplication of (28) by $A x$ yields

$$
\begin{array}{r}
P_{1} A x=\left[\left(a_{1} \bar{x}_{e}+1\right) a_{1} x,\left(a_{2} \bar{x}_{e}+1\right) a_{2} x, \ldots,\right. \\
\left.\left(a_{n} \bar{x}_{e}+1\right) a_{n} x\right]^{T} .
\end{array}
$$


Using (26), (29) and (30), we obtain

$$
\begin{aligned}
x^{T} & \left(A^{T} P A-P\right) x \\
= & x^{T}\left(A^{T} P_{1}^{T} P_{1} A-P_{1}^{T} P\right) x \\
= & {\left[P_{1} A x\right]^{T}\left[P_{1} A x\right]-\left[P_{1} x\right]^{T}\left[P_{1} x\right] } \\
= & {\left[\left(a_{1} \bar{x}_{e}+1\right) a_{1} x,\left(a_{2} \bar{x}_{e}+1\right) a_{2} x, \ldots,\left(a_{n} \bar{x}_{e}+1\right) a_{n} x\right] } \\
& \times\left[\left(a_{1} \bar{x}_{e}+1\right) a_{1} x,\left(a_{2} \bar{x}_{e}+1\right) a_{2} x, \ldots,\left(a_{n} \bar{x}_{e}+1\right) a_{n} x\right]^{T} \\
- & {\left[\left(a_{1} \bar{x}_{e}+1\right) x_{1},\left(a_{2} \bar{x}_{e}+1\right) x_{2}, \ldots,\left(a_{n} \bar{x}_{e}+1\right) x_{n}\right] } \\
& \times\left[\left(a_{1} \bar{x}_{e}+1\right) x_{1},\left(a_{2} \bar{x}_{e}+1\right) x_{2}, \ldots,\left(a_{n} \bar{x}_{e}+1\right) x_{n}\right]^{T} \\
= & {\left[\sum_{i=1}^{n}\left(a_{i} \bar{x}_{e}+1\right)^{2}\left(\left(a_{i} x\right)^{2}-x_{i}^{2}\right)\right]<0, }
\end{aligned}
$$

since for an asymptotically stable 2D Roesser model we have

$$
\sum_{i=1}^{n}\left(a_{i} x\right)^{2}-x_{i}^{2}<0
$$

Example 2. Consider the positive asymptotically stable 2D Roesser model (10) with the matrix

$$
A=\left[\begin{array}{ll}
A_{11} & A_{12} \\
A_{21} & A_{22}
\end{array}\right]=\left[\begin{array}{cc:c}
0.1 & 0.3 & 0.2 \\
0 & 0.2 & 0.4 \\
\hdashline & \ldots & \ldots \\
0 & 0 & 0.3
\end{array}\right] .
$$

Using (15) and (27), we obtain

$$
\begin{aligned}
\bar{x}_{e} & =\left[I_{n}-A\right]^{-1} \mathbf{1}_{n}=\left[\begin{array}{ccc}
0.9 & -0.3 & -0.2 \\
0 & 0.8 & -0.4 \\
0 & 0 & 0.7
\end{array}\right]^{-1}\left[\begin{array}{l}
1 \\
1 \\
1
\end{array}\right] \\
& =\frac{1}{0.504}\left[\begin{array}{l}
1.05 \\
0.99 \\
0.72
\end{array}\right]
\end{aligned}
$$

and

$$
\begin{aligned}
P_{1} & =\operatorname{diag}\left[\bar{x}_{e 1}, \bar{x}_{e 1}, \bar{x}_{e 3}\right] \\
& =\frac{1}{0.504}\left[\begin{array}{ccc}
1.05 & 0 & 0 \\
0 & 0.99 & 0 \\
0 & 0 & 0.72
\end{array}\right] .
\end{aligned}
$$

The strictly positive diagonal matrix $P$ has the form

$$
\begin{aligned}
P & =P_{1}^{T} P_{1} \\
& =\frac{1}{(0.504)^{2}}\left[\begin{array}{ccc}
(1.05)^{2} & 0 & 0 \\
0 & (0.99)^{2} & 0 \\
0 & 0 & (0.72)^{2}
\end{array}\right] .
\end{aligned}
$$

From (31) and (32) we have

$$
A^{T} P A-P=\left[\begin{array}{rrr}
-4.2969 & 0.1302 & 0.0868 \\
0.1302 & -3.3135 & 0.5691 \\
0.0868 & 0.5691 & -1.0662
\end{array}\right] \text {. }
$$
definite.

It is easy to check that the matrix (33) is negative

\section{Concluding Remarks}

The problem of choosing the forms of Lyapunov functions for the positive 2D Roesser model has been addressed. It has been shown that for the positive 2D Roesser model (i) the linear form of the state vector (8) can be chosen, as a Lyapunov function (ii) there exists a strictly positive diagonal matrix $P$ of the form (26) such that the condition (23) is satisfied. The deliberations were illustrated with two numerical examples. An extension of these ideas to a general positive 2D model remains an open problem.

\section{References}

Benvenuti L. and Farina L. (2004): A tutorial on the positive realization problem. IEEE Transactions on Automatic Control, Vol. 49, No. 5, pp. 651-664.

Bose N. K. (1985): Multidimensional Systems Theory Progress, Directions and Open Problems, Dordrecht: D. Reidel Publishing Co.

Farina L. and Rinaldi S. (2000): Positive Linear Systems. Theory and Applications. New York: Wiley.

Fornasini E. and Marchesini G. (1978): Double indexed dynamical systems. Mathematical Systems Theory, Vol. 12, pp. 59-72.

Fornasini E. and Marchesini G. (1976): State-space realization theory of two-dimensional filters. IEEE Transactions on Automatic Control, Vol. AC-21, pp. 484-491.

Fornasini E. and Valcher M.E. (1996): On the spectral and combinatorial structure of $2 D$ positive systems. Linear Algebra and Its Applications, Vol. 245, pp. 223-258.

Fornasini E. and Valcher M.E. (1997): Recent developments in $2 D$ positive systems theory. International Journal of Applied Mathematics and Computer Science, Vol. 7, No. 4, pp. 101-123.

Gałkowski K. (1997): Elementary operation approach to state space realization of $2 D$ systems. IEEE Transaction on Circuits and Systems, Vol. 44, No. 2, pp. 120-129.

Kaczorek T. (1999): Externally positive 2D linear systems. Bulletin of the Polish Academy of Sciences: Technical Sciences, Vol. 47, No. 3, pp. 227-234.

Kaczorek T. (1996): Reachability and controllability of nonnegative 2D Roesser type models. Bulletin of the Polish Academy of Sciences: Technical Sciences, Vol. 44, No. 4, pp. 405-410. 
Kaczorek T. (2000): Positive $1 D$ and 2D Systems. London: Springer.

Kaczorek T. (2002): When the equilibrium of positive $2 D$ Roesser model are strictly positive. Bulletin of the Polish Academy of Sciences: Technical Sciences, Vol. 50, No. 3, pp. 221-227.

Kaczorek T. (1985): Two-Dimensional Linear Systems. Berlin: Springer.

Klamka J. (1999): Controllability of 2D linear systems, In: Advances in Control Highlights of ECC 1999 (P.M. Frank, Ed.), Berlin: Springer, pp. 319-326.

Klamka J. (1991): Controllability of dynamical systems. Dordrecht: Kluwer.

Kurek J. (1985): The general state-space model for a twodimensional linear digital systems. IEEE Transactions on Automatic Control, Vol. -30, No. 2, pp. 600-602.
Kurek J. (2002): Stability of positive $2 D$ systems described by the Roesser model. IEEE Transactions on Circuits and Systems I, Vol. 49, No. 4, pp. 531-533.

Roesser R.P. (1975) A discrete state-space model for linear image processing. IEEE Transactions on Automatic Control, Vol. AC-20, No. 1, pp. 1-10.

Valcher M.E. and Fornasini E. (1995): State models and asymptotic behaviour of $2 D$ Roesser model. IMA Journal on Mathematical Control and Information, No. 12, pp. 17-36.

Received: 25 June 2007

Revised: 30 July 2007 
